\title{
Angoisse existentielle, angoisse pathologique
}

\section{Philippe Cabestan}

\section{OpenEdition}

\section{Journals}

Édition électronique

URL : https://journals.openedition.org/alter/1897

DOI : $10.4000 /$ alter.1897

ISSN : 2558-7927

\section{Éditeur :}

Association ALTER, Archives Husserl (CNRS-UMR 8547)

\section{Édition imprimée}

Date de publication : 1 novembre 2019

Pagination : 121-138

ISBN : 978-2-9550449-5-7

ISSN : 1249-8947

Référence électronique

Philippe Cabestan, « Angoisse existentielle, angoisse pathologique », Alter [En ligne], 27 | 2019, mis en ligne le 22 décembre 2020, consulté le 13 juin 2021. URL : http://journals.openedition.org/alter/1897 ; DOI : https://doi.org/10.4000/alter.1897

Ce document a été généré automatiquement le 13 juin 2021.

Revue Alter 


\title{
Angoisse existentielle, angoisse pathologique
}

\author{
Philippe Cabestan
}

1 Quelques années avant sa mort, Sartre soutient que, même s'il lui est arrivé d'écrire sur de tels sujets, il ignore aussi bien l'angoisse que le désespoir. Avec la sincérité - semblet-il - d'un homme délivré du qu'en-dira-t-on, l'auteur de L'Être et le néant déclare : «j'ai parlé de désespoir, mais c'est de la blague, j'en ai parlé parce qu'on en parlait, parce que c'était la mode : on lisait Kierkegaard », et il ajoute à propos de l'angoisse : «je n'ai jamais eu d'angoisse. Ce sont des notions clefs de la philosophie de 1930 à 1940. Cela venait aussi de Heidegger, c'est des notions dont on se servait tout le temps, mais qui ne correspondaient pour moi à rien $»^{1}$. Reconnaissons que ces propos sont embarrassants et, ce, d'autant plus que L'Être et le néant accorde une place décisive à l'angoisse qui est pour le sujet sartrien la conscience même de sa liberté ${ }^{2}$. À vrai dire, la déclaration de Sartre a le mérite de nous mettre en garde en nous rappelant une dimension fondamentale de l'angoisse: son absence d'évidence. Non pas au sens technique que ce terme revêt chez Descartes ou Husserl mais au sens simplement de sa relative et déroutante indétermination, tout du moins pour un certain nombre d'entre nous. En d'autres termes, alors que chacun sait ce qu'est la colère, la joie ou la tristesse, l'angoisse résiste à qui veut s'en emparer au point que nul ne semble en mesure de distinguer en toute rigueur l'angoisse, de la peur, de la crainte, de l'effroi, de l'anxiété ou de la détresse. On sait du reste que la langue allemande, par exemple, ne distingue pas nettement entre la peur et l'angoisse et l'expression Angst haben peut être traduite aussi bien par « avoir peur " que par « éprouver de l'angoisse ». De même, en anglais, le mot anxiety peut être traduit selon les circonstances par anxiété, inquiétude ou angoisse ${ }^{3}$. Ainsi que signifie précisément être angoissé ?

2 L'angoisse est incontestablement une notion des plus courantes. On dit parfois qu'on est angoissé pour désigner un simple malaise dont on dira après coup que ce n'était rien. Elle peut prendre la forme de violentes crises qui se terminent dans les larmes. On sait l'importance que Freud et ses successeurs lui ont accordée. De fait, l'angoisse est l'objet en psychanalyse d'une élaboration théorique toute particulière et se décline sous 
les formes de la phobie ou hystérie d'angoisse, de la névrose d'angoisse, du signal d'angoisse, de l'angoisse de castration, etc. Elle est par ailleurs au centre du cas clinique, évoqué par Binswanger et sur lequel nous reviendrons, d'une jeune fille qui est " assaillie d'une angoisse indomptable » lorsqu'elle remarque qu'un talon ne tient pas solidement à la chaussure. De son côté, le Dictionnaire de psychiatrie et de psychopathologie clinique, tout en reconnaissant que la distinction entre angoisse et anxiété est difficile à faire, propose la définition qui suit: « Ensemble de sentiments et de phénomènes affectifs caractérisé par une sensation interne d'oppression et de resserrement et par la crainte réelle ou imaginaire d'un malheur grave ou d'une grande souffrance devant lesquels on se sent à la fois démuni et totalement impuissant à se défendre ». Cette définition s'appuie à sa manière sur l'étymologie qui nous apprend que le mot angoisse vient du latin angustus qui signifie : étroit, resserré. Ainsi l'angoisse n'est-elle pas un phénomène purement psychique mais présente une dimension somatique et neurovégétative: constriction œsophagienne, douleurs thoraciques (précordialgie), difficultés respiratoires (dyspnée), tachycardie, tremblements, hypersudation, diarrhée, etc. ${ }^{4}$

3 Ces considérations préliminaires nous conduisent ainsi à nous interroger non seulement sur l'angoisse en tant que disposition affective mais en outre sur sa parenté avec l'angoisse en tant que trouble psychopathologique. Autrement dit, l'angoisse éprouvée par un agoraphobe est-elle assimilable à celle que peut connaître tout un chacun ou bien est-elle de nature profondément différente? Et si l'angoisse, comme nous allons le voir, est à la source selon Heidegger d'un mode d'existence authentique, quelle est la nature de cette angoisse pathologique que Michel Haar, pour sa part, qualifie d'inauthentique ${ }^{5}$ ? Afin de répondre à ces questions, nous voudrions tout d'abord envisager les différentes descriptions de l'angoisse d'un point de vue phénoménologique. Puis, nous rappellerons brièvement les grandes lignes de la théorie freudienne de l'angoisse. Enfin, à partir de l'analyse de deux ou trois cas, nous essayerons de déterminer en quel sens la phobie est synonyme d'angoisse.

\section{L'angoisse en tant que disposition fondamentale}

Le concept d'angoisse tel que nous allons le développer est, de fait, étroitement lié à un certain type de philosophies que l'on peut appeler les philosophies de l'existence. Sans doute trouve-t-on chez Aristote une analyse des différentes passions humaines et, entre autres, de la crainte (phobos). Mais il semble que Aristote ignore l'angoisse en tant que telle (quand bien même, soutient Heidegger en 1924, il aurait frôlé le phénomène de l'angoisse à propos de la mort) ${ }^{6}$. Lorsque Heidegger soutient: "le phénomène de l'angoisse n'est pas une trouvaille de ma part ", et qu'il s'interroge sur les analyses qui ont pu précéder la sienne, il cite saint Augustin, Luther dans son commentaire de la Genèse et Kierkegaard ${ }^{7}$. Cela dit, on peut distinguer deux grandes conceptions de l'angoisse, qui ne nous semblent nullement contradictoires dans la mesure où elles sont relatives à l'être de l'homme en tant qu'être-au-monde, et que nous allons envisager successivement. D'une part, la conception heideggérienne qui tient l'angoisse pour une disposition affective fondamentale (Grundstimmung), qui révèle au Dasein son mode d'être et dont l'analyse phénoménologique occupe un rôle décisif dans l'économie de l'ontologie fondamentale; d'autre part, la conception kierkegaardienne et sartrienne qui envisage l'angoisse comme «le vertige de la liberté ». Parce que ces analyses sont 
déjà plus ou moins connues, nous nous contenterons ici d'en rappeler les traits essentiels.

Selon Heidegger, l'angoisse est une disposition fondamentale du Dasein de telle sorte qu'exister et être angoissé sont une seule et même chose. Un concours de circonstances exceptionnelles n'est donc pas nécessaire pour qu'elle survienne. Il suffit, au contraire, d'un événement anodin pour que, sans crier gare, l'angoisse s'insinue voire s'empare du Dasein. Il va de soi que cette circonstance n'est jamais que l'occasion du surgissement de l'angoisse et non sa cause. De même, si l'angoisse présente une évidente dimension physiologique, elle ne saurait avoir une simple origine physiologique, comme le voudrait une approche naturaliste de ce type de phénomènes. Heidegger souligne précisément que «le déclenchement physiologique de l'angoisse n'est possible que parce que le Dasein, dans le fond de son être, s'angoisse ». Le Dasein, en effet et en dépit des apparences, ne cesse d'être angoissé. Simplement, il se masque l'angoisse qui l'habite en s'oubliant dans de multiples préoccupations qui relèvent de ce mode d'existence qu'Etre et temps tient pour inauthentique. Cette fuite permanente du Dasein peut rappeler le divertissement au sens pascalien du terme ${ }^{8}$. Elle s'en distingue cependant dans la mesure où le libertin pour Pascal fuit la misère d'une existence sans Dieu, tandis que selon Heidegger, c'est l'existence en tant que telle qui est la source de l'angoisse et que fuit le Dasein. Heidegger reprend à cette occasion le concept théologique de chute (Verfallenheit) tout en lui donnant une signification différente : la chute désigne la fuite du Dasein dans ce monde familier que régit la puissance anonyme du On et, ce, afin de se soustraire à l'angoisse qui le hante.

6 Mais afin de mieux saisir cette conception de l'angoisse, il convient de distinguer - avec Heidegger mais aussi le psychologue français Pierre Janet ${ }^{9}$ - la peur et l'angoisse ${ }^{10}$. La peur (Furcht), comme la colère ou la frayeur, est une émotion qui se rapporte à un objet. Elle est toujours peur de quelque chose, c'est-à-dire d'un étant quelconque qui apparaît ici ou là et qui nous menace d'une manière ou d'une autre. De son côté, l'angoisse n'a pas à proprement parler d'objet. En effet, ce devant-quoi l'angoisse est angoisse n'est pas un étant déterminé comme un chien qui gronde ou un précipice; ce n'est pas quelque chose qui se trouve dans le monde et qui, par suite, se trouverait ici ou là-bas. Mais ce devant-quoi l'angoisse s'angoisse, c'est l'être-au-monde lui-même: dans l'angoisse, le Dasein se rapporte à la totalité de l'étant et découvre un monde qui surgit dans son étrangeté (Unheimlichkeit). Une telle expérience n'a rien d'extraordinaire et on peut rappeler à ce propos la sentence de Pascal: «Le silence éternel de ces espaces infinis m'effraie »; ou le vers non moins célèbre de Lamartine: «Un seul être vous manque, et tout est dépeuplé ». L'effroi pascalien ou la solitude du poète romantique, par-delà la singularité de leurs perspectives, renvoient en effet à une même expérience fondamentale de déréliction (Geworfenheit) que l'on peut également rapprocher de l'état de détresse ou désaide (Hilflosigkeit) du nourrisson ${ }^{11}$. Ainsi la fuite du Dasein est-elle, comme l'écrit Heidegger, "fuite devant le pas-chez-soi, c'est-à-dire devant l'étrangeté inhérente au Dasein en tant qu'être-au-monde jeté, livré en son être à lui-même $»^{12}$. Et c'est grâce à ses préoccupations quotidiennes que le Dasein retrouve un monde qui lui est familier.

7 Toutefois, au sein même de ce monde familier, la mort et tout ce qui s'y rapporte constituent une occasion privilégiée pour rappeler au Dasein l'angoisse qui le hante. Remarquons qu'il faut à nouveau ne pas confondre l'angoisse et la peur, et que l'angoisse face à la mort n'est pas à proprement parler la peur de la mort. Celle-ci est 
relative à l'événement du décès et à ses multiples modalités possibles. Elle est liée à des questions existentielles telles que: Vais-je souffrir? Cela durera-t-il longtemps? Qu'est-ce qui m'attend après ? Celle-là a une signification proprement ontologique dans la mesure où elle étreint le Dasein en lui dévoilant son être ${ }^{13}$. Sans doute le Dasein a-t-il la possibilité de se dissimuler sa mortalité derrière un rassurant "on meurt", qui concerne à la fois tout le monde et personne en particulier ${ }^{14}$. Il peut également s'adresser à la métaphysique afin de mieux se persuader de son immortalitée ${ }^{15}$. Mais, ce faisant, il se condamne à se masquer son être en tant qu'être jeté vers sa fin ${ }^{16}$. C'est pourquoi, Heidegger recommande au Dasein de ne pas fuir l'angoisse mais de l'affronter résolument. L'angoisse apparaît alors comme la condition de l'authenticité (Eigentlichkeit) : elle isole au sens où, comme nous allons le voir, elle singularise le Dasein, et «accomplit cette modification existentielle par laquelle le soi comme On se transforme en soi "authentique" $»^{17}$.

\section{Kierkegaard, Sartre ou l'angoisse de la liberté}

8 La conception heideggérienne de l'angoisse se réfère explicitement à Kierkegaard, même si elle ne s'inscrit pas dans le contexte théologique qui est celui du philosophe danois. Car l'angoisse pour Kierkegaard est étroitement liée à l'innocence, au péché originel et à la chute au sens biblique du terme. C'est du reste la raison pour laquelle l'analyse de Kierkegaard dans le Concept d'Angoisse (1844) est centrée sur les figures d'Adam et Eve. Cependant, si nous essayons de rapprocher les conceptions tant heideggériennes que kierkegaardiennes de l'angoisse, il est relativement facile d'en dégager un noyau commun, même si elles ne se confondent pas.

9 Tout d'abord, loin de tenir l'angoisse pour un phénomène essentiellement pathologique, Kierkegaard considère comme Heidegger que tout homme est angoissé, y compris le plus heureux des hommes s'il existe. En outre, Kierkegaard et Heidegger tiennent à ne pas confondre l'angoisse qui ne se rapporte à rien d'étant, et la peur ou la crainte et ce qui leur ressemble ${ }^{18}$. Enfin et surtout, l'angoisse est pour l'un comme pour l'autre une épreuve de la liberté. En effet, alors que la quotidienneté de la préoccupation selon Heidegger est "aveugle au possible (möglichkeitsblind)» et se satisfait du simplement "effectif", il revient à l'angoisse de mettre le Dasein devant ses propres possibilités ${ }^{19}$. Ainsi non seulement l'angoisse esseule, c'est-à-dire ramène le Dasein à lui-même mais, en outre, elle dévoile au Dasein sa liberté, c'est-à-dire son "l'être-libre pour la liberté de se choisir et de se saisir soi-même " ${ }^{20}$. En d'autres termes, que nous empruntons à Jean Greisch, "sans angoisse, pas de liberté et réciproquement $! »^{21}$. De fait, nous retrouvons chez Kierkegaard une conception analogue de l'angoisse qui s'apparente au vertige, c'est-à-dire à cette étrange attraction et répulsion qu'on éprouve quand on plonge le regard dans un abîme. Et "l'objet" comme le "sujet" de cette angoisse est la liberté. Plus précisément, avec l'interdiction divine, naît la tentation. La liberté s'éveille alors et ce pouvoir de transgresser l'interdiction divine est à la fois ce qui attire Adam et le repousse de telle sorte que l'angoisse est décrite par Kierkegaard en termes de sympathie et d'antipathie. Ainsi Kierkegaard peut-il définir l'angoisse comme « le vertige de la liberté »².

Nous retrouvons mutatis mutandis cette conception de l'angoisse dans L'Être et le néant qui entend conjuguer l'approche kierkegaardienne de l'angoisse comme angoisse devant la liberté et l'approche heideggérienne, développée notamment dans la 
conférence de 1929, Qu'est-ce que la métaphysique?, de l'angoisse comme saisie du néant : "ces deux descriptions de l'angoisse, écrit Sartre, ne nous paraissent pas contradictoires : elles s'impliquent l'une l'autre au contraire $»^{23}$. Aussi Sartre reprend-il à sa manière la situation paradigmatique du vertige qui saisit le sujet au bord d'un précipice. Comme Kierkegaard, Sartre distingue entre la peur de tomber dans le précipice qui représente un danger de mort et le vertige en tant que tel : «Le vertige est angoisse dans la mesure où je redoute non de tomber dans le précipice mais de m'y jeter ». Apparaît cependant ce qui distingue l'analyse sar-

trienne. L'angoisse n'est pas pour Sartre ce mélange d'attirance et de répulsion qui conduit Adam et Eve aux portes du péché, mais la conscience qu'il m'est possible de m'écarter du précipice comme de m'y précipiter. En constituant une conduite comme ma conduite possible, «je me rends compte que rien ne peut m'obliger à tenir cette conduite ». Rien, car cette conduite échappe à l'enchaînement naturaliste des causes et des effets ; elle n'est jamais qu'une conduite future possible qui est séparée par un néant de mon être présent. En ce sens, l'angoisse sartrienne révèle à la conscience son mode d'être, c'est-à-dire le néant qui l'habite et qui fonde ontologiquement sa liberté.

Lorsque Sartre soutient que «ces deux descriptions de l'angoisse ne nous paraissent pas contradictoires » et même qu'elles "s'impliquent l'une l'autre au contraire », il ne faut sans doute pas prendre l'affirmation au pied de la lettre : il va de soi que le rien ou néant sartrien dans son opposition antithétique à l'être est distinct du rien qui ne fait qu'un avec l'étant de la conférence Qu'est-ce que la métaphysique ?24 On peut noter en outre qu'en focalisant sa description de l'angoisse sur la liberté, Sartre néglige certains traits de l'angoisse heideggérienne et, notamment, la déréliction du Dasein. Cependant, par-delà ce qui les sépare, ces différentes conceptions nous permettent de reconnaître dans l'angoisse une disposition affective fondamentale (Grundstimmung), indépendante d'un objet déterminé, qui se rapporte à l'être de l'homme, en tant que liberté jetée dans un monde qui lui est étranger (unheimlich). Nous pouvons alors reposer notre question : cette conception de l'angoisse permet-elle d'éclairer certaines dispositions pathologiques qui, du moins en apparence, s'y apparentent au point qu'on les désigne à l'aide d'un seul et même terme? Cependant, il nous faut auparavant brièvement évoquer la ou plutôt les conceptions freudiennes de l'angoisse auxquelles on se réfère habituellement en psychopathologie.

\section{Les conceptions freudiennes de l'angoisse}

Difficile, en effet, d'aborder la question de l'angoisse en phénoménonologie et en psychopathologie sans dire ne serait-ce qu'un mot de la conception freudienne de l'angoisse. Non seulement parce qu'elle a durablement marqué les esprits mais aussi parce qu'elle est à certains égards contestable - nous laisserons ici volontairement de côté ses "prolongements" relativement obscurs dans le livre X du Séminaire de Lacan ${ }^{25}$.

D'une manière très générale, si nous voulons élucider l'angoisse en tant que disposition affective, il convient tout d'abord de se tenir à distance d'une conception réifiante ou chosiste de l'affectivité qui domine la manière dont on l'aborde bien souvent - en prétendant par exemple mesurer l'intensité d'un sentiment - et que l'on retrouve chez Freud et ses héritiers. Pour ce qui concerne ce dernier, il suffit de réfléchir sur la dénomination même de transfert (Übertragung), ou sur celle de déplacement (Verschiebung), pour saisir immédiatement la chosification de ce que Freud appelle 
affect : comme s'il était possible de transférer un sentiment comme on transfère les cendres d'un homme illustre. Medard Boss écrit à ce propos :

Par le concept de transfert aussi, Freud a certes fait référence à un phénomène déterminant de tout traitement médical. Pourtant l'interprétation qu'il en a déjà donnée à travers cette appellation a au fond comme présupposition qu'il y a pour ainsi dire des sentiments en soi, détachés des choses senties ou des êtres humains perçus. Car seule l'hypothèse de sentiments isolés à la façon des choses et subsistant de manière autonome permet d'imaginer que, par exemple, un sentiment de haine puisse être détaché d'un père et, au cours d'une psychanalyse, être transféré sur l'analyste ${ }^{26}$.

14 Sans doute est-il légitime d'interroger la parenté entre l'attitude d'un patient vis-à-vis de son père, de son analyste et de la loi en général, mais parce que la haine est un sentiment, mieux: une disposition affective envers une personne qu'elle dévoile comme haïssable, elle ne saurait en tant que telle faire "l'objet" d'un quelconque transport ou déplacement de sorte que, selon la logique de l'identité substantielle, il s'agirait par-delà les apparences de la même haine quoi qu'elle vise une autre personne dans des circonstances temporelles distinctes.

Outre cette objection de principe, la manière même dont Freud envisage l'angoisse est également contestable. Afin de clarifier la discussion, il convient cependant de rappeler brièvement que, à la suite de Jean Laplanche ${ }^{27}$, on peut distinguer deux théories de l'angoisse dans l'œuvre de Freud. D'une part, une théorie physiologique et économique, exposée en 1916 dans l'Introduction à la psychanalyse, selon laquelle l'origine de l'angoisse, comme le révélerait en particulier l'angoisse névrotique, est sexuelle. Il y aurait "une relation génétique entre la libido et l'angoisse " même si, comme le reconnaît Freud, « on ne voit pas tout d'abord comment l'angoisse naît de la libido ${ }^{28}$. Pour tenter d'élucider malgré tout cette mystérieuse transmutation, Freud compare l'angoisse à du vinaigre et soutient alors que l'angoisse serait un produit de la libido comme le vinaigre l'est $\mathrm{du} \mathrm{vin}^{29}$. Conscient le tout premier des faiblesses d'une telle explication, Freud remanie en profondeur sa première théorie de l'angoisse et, en 1920, dans Inhibition, symptôme et angoisse, rapporte l'angoisse non plus à la libido mais au moi. Désormais, comme il l'écrit, «c'est l'angoisse qui fait le refoulement et non pas, comme je l'ai estimé jadis, le refoulement qui fait l'angoisse $»^{30}$. En d'autres termes, c'est l'angoisse qui conduit le moi à refouler des pulsions sexuelles dangereuses, contrairement à l'idée qui était auparavant celle de Freud et selon laquelle la libido refoulée se transformait en angoisse. De ce point de vue, l'angoisse qui étreint le petit Hans est une angoisse de castration (déformée) qui signale le danger interne que représentent ses désirs incestueux. On voit ainsi comment cette deuxième conception de l'angoisse privilégie le modèle de ce que Freud appelle « l'angoisse de réel », c'est-àdire la peur éprouvée face à un danger extérieur. L'angoisse névrotique devient une réaction du moi face à un danger interne. En outre, cette deuxième conception, conformément à l'idée que les affects sont d'une manière générale des reproductions d'événements anciens, envisage l'angoisse comme la répétition d'un ensemble de phénomènes physiologiques qui accompagne la naissance.

Si cette deuxième conception de l'angoisse a le mérite de ne plus tenter une improbable dérivation de l'angoisse à partir de la libido, elle échoue néanmoins à distinguer entre la peur et l'angoisse et à ressaisir la signification existentielle voire ontologique de l'angoisse. On dira non sans raison que telle n'est pas l'ambition de Freud dont l'objet est avant tout l'angoisse pathologique. Le défi pour la phénoménologie est donc non 
seulement d'offrir une description plus rigoureuse de l'angoisse mais également de rendre de compte de l'angoisse en tant que phénomène pathologique. Ne faut-il pas donner raison à Freud lorsqu'il rapproche la peur face à un danger réel et l'angoisse pathologique?

\section{De l'effondrement du monde dans la phobie}

17 Si les êtres humains se soustraient tout d'abord et le plus souvent à l'empire de l'angoisse en se laissant happer par leurs différentes préoccupations ou en adoptant des conduites de mauvaise foi au sens sartrien du terme, force est de constater que certains ne "bénéficient" manifestement pas de cette possibilité au point que l'angoisse hypothèque gravement leur existence. Kimura Bin, psychiatre et philosophe japonais, lecteur et traducteur de Binswanger, écrit à ce propos: «Dans tous les troubles psychopathologiques on retrouve l'angoisse comme phénomène commun. Elle représente l'indice le plus essentiel des crises de l'existence humaine et des ébranlements psychiques ${ }^{31}$. Une semblable affirmation soulève une double question : la première, que nous connaissons déjà, concerne le lien éventuel entre l'angoisse que décrivent les philosophies de l'existence et l'angoisse pathologique qu'éprouvent certains malades. La seconde est de savoir si, selon les troubles en question: schizophrénie, mélancolie, hystérie, névrose obsessionnelle ou névrose de contrainte, etc., il s'agit à chaque fois de la même forme d'angoisse. Nous nous limiterons ici à l'angoisse dans la phobie que nous pouvons tout d'abord envisager à partir du cas présenté par Binswanger d'une phobie hystérique du talon ${ }^{32}$.

Il s'agit d'une jeune femme de vingt et un ans qui, dans sa cinquième année, alors qu'elle détachait ses patins, avait eu le talon de sa chaussure coincé dans le patin. Depuis, cette jeune femme est assaillie d'une angoisse (Angst) irrépressible, au point de s'évanouir si elle ne peut pas s'enfuir, lorsqu'elle remarque qu'un talon ne tient pas solidement à la chaussure, que quelqu'un se touche le talon, ou parle simplement d'un talon. Cependant, est-il adéquat dans le cas de cette jeune femme de parler d'angoisse? Phobie et angoisse sont à première vue bien distinctes : l'angoisse, avons-nous répété, est sans objet alors que la phobie est toujours la peur ou la crainte de quelque chose tel que la rupture du talon. Plus précisément, la phobie est une peur dite pathologique dans la mesure où elle est disproportionnée et sans fondement véritable, contrairement à la peur suscitée par l'apparition d'un animal féroce ou la vue d'un précipice. Toutefois, n'accordons-nous pas trop d'importance à l'objet au point d'occulter l'angoisse éprouvée par le malade à l'occasion de cet objet phobique ?

Disciple de Freud, Binswanger commence par mettre de côté l'explication à partir de ce qui s'était produit dans la cinquième année: loin d'être la cause explicative de la phobie, l'événement de la rupture du talon est «innocent en soi ». Binswanger fait sienne, en effet, la thèse freudienne selon laquelle ce sont les fantasmes qui se rattachent à un tel événement ou le précèdent, qui agissent de façon pathogène. Mais parce qu'elle ne permet pas de rendre compte pourquoi « l'événement ou le fantasme en question, précisément chez cette personne-là, a eu ces conséquences-là », cette analyse demande à être approfondie. Il convient alors de ne pas s'en tenir aux phantasmes et de remonter derrière les phantasmes jusqu'à ce que Binswanger dénomme le "projet-de-monde (Weltentwurf)" de la jeune femme ${ }^{33}$. Ce concept, qui peut rappeler celui de choix originel dans L'Être et le néant ${ }^{34}$, désigne la manière dont les 
étants, les hommes aussi bien que les choses, se dévoilent. Il va de soi, en effet, qu'un chêne n'a pas le même sens selon le monde et le projet de monde dans lequel il s'inscrit et que le chêne d'un chasseur n'est pas celui d'une jeune femme romantique ou d'un marchand de bois prosaïque ${ }^{35}$. D'un point de vue psychopathologique, il est également possible de distinguer les mondes et les projets-de-mondes du maniaque ou du schizophrène : «Étudier et établir le monde de ces malades signifie, ici comme ailleurs, étudier et établir de quelle manière l'étant devient accessible à ces formes d'êtreprésent (Dasein) $»^{36}$.

Pour Binswanger, on peut certes montrer d'un point de vue psychanalytique que derrière le talon branlant ou détaché se cachent des fantasmes de naissance et de séparation d'avec la mère qui impliquent une rupture de continuité entre la mère et l'enfant. Mais ces fantasmes comme la phobie du talon doivent être rapportés au projet-de-monde de cette jeune femme, dont le fil conducteur est la catégorie de la continuité. Binswanger écrit: "Tout ce qui rend le monde signifiant tombe sous la domination de cette seule catégorie. C'est elle seule qui confère au monde, et à l'être en lui, leur assise. De là, l'angoisse devant toute rupture de continuité $\|^{37}$. Comprenons : si la séparation d'avec la mère prend chez cette jeune femme une importance telle que tout événement de séparation, aussi anodin soit-il en apparence, en vient à la symboliser, ce n'est donc pas à cause d'un attachement œdipien originaire à la mère mais parce que cet attachement lui-même et son caractère excessif trouvent leur source dans un projet-de-monde restreint à la notion de continuité qui, nous reprenons délibérément la phrase de Binswanger, « seule confère au monde, et à l'être en lui, leur assise $\aleph^{38}$. Ainsi, dans le monde de cette jeune femme hystérique, toute solution ou rupture de continuité met le monde en crise et devient une expérience traumatique.

D'une manière générale, comme l'écrit Binswanger, l'angoisse survient «lorsque le "monde" se met à chanceler ou menace de disparaître (wenn die "Welt" ins Wanken gerät oder zu verschwinden droht) $»^{39}$. De ce point de vue, ce qui distingue l'homme sain de l'homme qui est en proie à des crises d'angoisse, c'est que son monde est suffisamment bien établi pour ne pas risquer de s'écrouler à l'occasion de tel ou tel événement qui n'en ébranle qu'un aspect. En revanche, le monde du malade est un monde appauvri en fonction d'un projet-de-monde pathologique et, par suite, particulièrement fragile. Comme nous l'avons vu, dans le cas de la phobie du talon rompu, le monde est vidé ou restreint parce qu'il est ressaisi à partir de la seule catégorie de la continuité. Il n'a pas cette richesse faite de la multiplicité des connexions et des rapports de sens. Phobie, écrit Binswanger, «[...] signifie toujours essai d'assurer un "monde" restreint, appauvri ; angoisse signifie perte de cette sécurisation, effondrement du "monde", et donc être-livré du Dasein au rien, à l'intenable et terrible "horreur nue" (Ausgeliefertsein des Daseins an des Nichts, an das unaushaltbare, fürchterliche "nackte Grauen")». Remarquons que cette conception de la phobie en tant que conduite permettant de prévenir un possible accès d'angoisse n'est pas sans rapport avec celle de Freud. Binswanger évoque explicitement la distinction freudienne entre la phobie comme symptôme et la libido comme véritable objet de l'angoisse. Même si ce qui pour lui est en jeu dans l'angoisse est tout autre qu'un objet, fût-il la libido, puisqu'il s'agit du rapport du malade au monde.

Cette idée d'un monde qui menace de s'effondrer renvoie explicitement à Heidegger et, plus précisément, à la conception développée en 1929 dans la conférence, Qu'est-ce que la métaphysique? où le devant-quoi de l'angoisse est alors non plus tant l'être-au-monde 
que le rien, le monde réduit à rien, si l'on peut dire. "L'angoisse, déclare Heidegger, manifeste le rien (Nichts) $»^{40}$. De même, pour Binswanger, le malade fait dans l'angoisse l'expérience fondamentale de l'indifférenciation de l'étant. Ainsi, contrairement aux apparences, la phobie ne doit pas être confondue avec la peur d'un quelconque danger mais elle est une angoisse pathologique qui, confrontée à la possibilité d'un effondrement du monde, n'est pas fondamentalement différente de l'angoisse en tant que disposition du Dasein. Cependant, elle s'en distingue à un double titre : d'une part, en fonction de ses conditions d'apparition liées à un projet-de-monde pathologique ; d'autre part, en raison de son inauthenticité. En effet, comme l'écrit Binswanger à propos d'une de ses patientes, dont il publie le cas en 1949, tandis que l'angoisse dévoile la possibilité d'un pouvoir-être authentique, "Lola reste jetée dans l'angoisse, sans aucune possibilité de se récupérer elle-même à partir d'elle $»^{41}$. En ce sens, la phobie peut être qualifiée à bon droit d'angoisse inauthentique ${ }^{42}$. On voit ainsi comment Binswanger, le psychiatre, fait sienne la conception heideggérienne de l'angoisse tout en la réélaborant d'un point de vue psychopathologique alors que, soulignons-le, la perspective de Heidegger est strictement commandée par la question de l'être ou Seinsfrage ${ }^{43}$.

\section{Phobie et vertige de la liberté}

Mais la phobie peut également être étroitement liée à la liberté et à ce vertige de la liberté que nous avons analysé avec Kierkegaard et Sartre. C'est ce que nous pouvons montrer à partir de la brève analyse de deux cas. Le premier est évoqué à la fin de la Transcendance de l'ego ${ }^{44}$ par Sartre, qui l'emprunte lui-même à l'ouvrage de Pierre Janet, Les Obsessions et la psychasthénie (1903). Il s'agit d'une jeune mariée qui avait la terreur quand son mari la laissait seule, de se mettre à la fenêtre et d'interpeller les passants à la façon des prostituées. Sartre écrit à son propos :

Rien dans son éducation, dans son passé, ni dans son caractère ne peut servir d'explication à une crainte semblable. Il nous paraît simplement qu'une circonstance sans importance (lecture, conversation, etc.) avait déterminé chez elle ce qu'on pourrait appeler un vertige de la possibilité. Elle se trouvait monstrueusement libre et cette liberté vertigineuse lui apparaissait à l'occasion de ce geste qu'elle avait peur de faire (nous soulignons).

Cette interprétation de ce qu'on pourrait appeler une phobie obsessionnelle peut non sans raison faire sourire. Selon Sylvie Le Bon, Sartre serait lui-même revenu sur son analyse en jugeant notamment « enfantine » sa mise entre parenthèses de l'éducation comme du passé et du caractère de la jeune mariée ${ }^{45}$. Toutefois, elle n'en mérite pas moins de retenir l'attention en raison de la manière dont elle reprend la notion kierkegaardienne de vertige et dont elle articule la peur, l'angoisse et l'obsession. En effet, il y a, occupant le premier plan, une peur excessive qui a pour objet une conduite qui ne sied absolument pas à une jeune mariée : se mettre à la fenêtre et interpeller les passants comme une vulgaire prostituée. Cette phobie doit pouvoir, au moins pour une part, se comprendre à partir de l'histoire de cette jeune mariée, de sa vie sexuelle et de ce que Binswanger appellerait son projet-de-monde. Mais par-delà cette conduite redoutée, l'analyse découvre l'angoisse qui n'a pas à proprement parler d'objet dans la mesure où elle est un vertige de la liberté, c'est-à-dire un vertige qui a pour "objet" et "sujet" la liberté. En tant que tel, ce vertige est indépendant de l'éducation comme du caractère pour la simple raison qu'il est constitutif de l'être de la conscience et peut 
être provoqué par " une circonstance sans importance ». Enfin, cette phobie présente un caractère obsessionnel que Sartre ressaisit à partir de l'idée - énigmatique - selon laquelle la conscience est susceptible de devenir captive d'elle-même, comme dans le cas où, ne voulant pas penser à tel événement, elle y pense précisément pour cela ${ }^{46}$. Quoi qu'il en soit, retenons qu'il est possible en droit de distinguer dans cette terreur de la jeune mariée ce qui relève de la phobie et de l'angoisse.

Le deuxième cas est tiré d'un article du psychiatre Alfred Kraus, consacré aux phobies dites spatiales, telles que la claustrophobie, l'agoraphobie ou encore phobie de la hauteur ou phobie de la chute. Dans cet article, inspiré de la lecture de la phénoménologie sartrienne, Kraus étudie «Les phobies de l'espace du point de vue de l'analyse existentielle et leur traitement $»^{47}$. Il insiste tout d'abord sur les transformations corporelles qui, d'une manière générale, accompagnent les émotions et qu'il ne faudrait pas séparer de la conscience comme de la conduite de la personne prise de phobie. Car ces modifications corporelles participent de la transformation du rapport de la conscience au monde. Plus précisément, et Alfred Kraus s'inspire ici explicitement de l'Esquisse d'une théorie des émotions de Sartre, dans la phobie comme dans l'émotion en général, le sujet se rapporte aux choses et au monde sur un mode magique et non plus sur un mode pragmatique ${ }^{48}$. Ainsi des relations nouvelles et fondamentalement irrationnelles s'établissent dans la phobie entre les choses et le sujet. Ces nouvelles relations se substituent aux relations objectives entre les objets et le sujet, dont les actions visent à transformer réellement le monde, et le monde est alors comme frappé de déréalisation. Par exemple, dans la phobie, la conscience perd toute distance non seulement vis-à-vis de ce qu'elle éprouve mais également vis-à-vis des objets auxquels elle se rapporte et qui la menacent. Dans la phobie un danger relativement éloigné perd son éloignement et la menace qu'il représente devient imminente et inévitable.

Pour illustrer son propos, Kraus reprend parmi d'autres cas celui d'un psychiatre qui souffre d'une phobie étroitement liée à la phobie de la hauteur et de la chute et qui est la phobie des voyages en avion. Ce psychiatre, Dörr-Zegers, a lui-même décrit la manière dont la phobie s'empare de lui et, notamment, comment tout à coup il n'est plus lui-même (dépersonnalisation) : «je ne suis plus celui que j'étais, ma famille, mon enfance, mon travail... tout a disparu ». Ses mains sont moites, il a l'impression d'avoir un trou dans le ventre, il éprouve un sentiment d'oppression dans la poitrine, ses jambes sont comme de plomb et il a le sentiment d'être un corps plutôt qu'un esprit. Contrairement aux autres passagers qui voyagent à ses côtés, il a perdu la capacité, ditil, d'appréhender l'avion comme s'il s'agissait d'une sorte de salon ou d'hôtel dans lequel il serait assis. Il se sent absolument seul et comme suspendu dans le vide sans pouvoir se raccrocher à quoi que ce soit : tout a disparu ${ }^{49}$. Nous retrouvons ainsi l'idée développée par Binswanger selon laquelle la phobie implique pour le malade une expérience angoissée du rien, au sens d'une dissolution des étants, et du dévoilement de l'être du Dasein en tant qu'être jeté dans un monde qui lui est étranger. Toutefois, Kraus ne s'en tient pas à une approche heideggérienne de la phobie et interroge ce vertige caractéristique de la phobie. Dans le cas de ce psychiatre qui souffre d'une phobie de l'avion, le vertige doit se comprendre comme un vertige de la liberté, au sens à la fois kierkegaardien et sartrien, vertige qui s'empare du sujet précisément au moment où il envisage de monter en avion et choisit ce faisant de risquer sa vie. Il ne s'agit pas en l'occurrence de la peur de mourir qui est un aspect de la phobie des avions 
mais de l'angoisse ou vertige d'une liberté qui a le choix entre risquer sa vie ou ne pas la risquer. Il est ainsi possible de distinguer dans la phobie, ce qui relève, d'une part, de la peur d'un accident mortel, de la peur de la mort et, d'autre part, de l'angoisse existentielle qui concerne l'être du sujet comme être-au-monde.

\section{Conclusion}

Lorsqu'il s'agit de l'angoisse, il convient d'être prudent. Car il n'y a en la matière rien d'évident, et nul n'est assuré de ne pas surinterpréter le phénomène en question ${ }^{50}$. Il semble cependant que l'on puisse tenir pour acquise l'idée que l'angoisse n'a pas d'objet, y compris d'objet intérieur tel que la libido, qu'elle est en tant que telle distincte de la peur, comme de la crainte ou de l'anxiété, et que ce qui y est en question n'est autre que l'être de l'homme. De ce point de vue, ne confondons pas la peur de la mort et l'angoisse liée à notre être mortel ou mortalité, même si dans les faits, lorsque nous sommes confrontés à la mort d'un proche, nous ne savons pas au juste pourquoi nous pleurons : outre la peur de la mort et la tristesse liée à la disparition d'un être cher, n'est-ce pas en effet sur nous-mêmes que nous pleurons, c'est-à-dire sur notre propre détresse au sein d'un monde proprement angoissant?

Sans doute reprochera-t-on à cette analyse de pratiquer un certain éclectisme et d'ignorer non seulement la perspective proprement ontologique de Heidegger mais aussi ce qui le sépare de Kierkegaard et de Sartre. Il va de soi que l'analyse de l'angoisse dans Être et temps comme dans Qu'est-ce que la métaphysique? - et Heidegger ne cesse de le répéter - n'est pas menée en vue d'une anthropologie et encore moins d'une psychopathologie phénoménologique. Dès lors, faire appel au concept d'angoisse afin d'élucider certains troubles pathologiques exige un remaniement de son concept qui ne peut être mécaniquement appliqué en dehors de la perspective ontologique pour laquelle il a été initialement élaboré. Comme le souligne Binswanger, l'angoisse pathologique n'a nullement la positivité de l'angoisse décrite dans Être et temps, en tant que disposition fondamentale qui dévoile à la liberté la possibilité de l'authenticité. L'angoisse pathologique se présente tout à l'inverse comme une angoisse inauthentique au sens où, comme l'écrit Heidegger à propos de la peur, elle est une disposition qui « ramène le Dasein à son être-jeté, mais de telle manière que celui-ci soit justement fermé $»^{51}$.

Enfin, l'analyse de certains troubles psychopathologiques conduit à réinterroger l'origine de l'angoisse, c'est-à-dire son devant-quoi qui, comme nous l'avons vu avec les cas de la phobie du vol en avion se rapporte aussi bien à l'effondrement du monde qu'à la liberté. On objectera peut-être qu'à vouloir conjuguer différentes approches de l'angoisse, on en vient à lui conférer un "objet" relativement indéterminé. Il serait cependant malheureux de vouloir spécialiser l'angoisse, et nous avons vu au contraire que l'angoisse précisément est relative à l'être-au-monde en tant que tel. D'un point de vue psychopathologique, il n'y a pas de raison de limiter l'angoisse au dévoilement du rien, sous prétexte que telle serait la thèse de Heidegger dans sa conférence de 1929. Comme le montre Kimura, l'angoisse peut se rapporter à d'autres aspects de l'être-aumonde, que ceux que nous avons jusqu'ici envisagés et notamment à sa temporalité : il y a, par exemple, dans la personnalité schizoïde une angoisse typique vis-à-vis de l'avenir tandis que la mélancolie est hantée par le passés5. Ainsi l'angoisse pathologique peut-elle prendre de multiples formes, dont l'exploration relève naturellement de la 
clinique. Elle n'en demeure pas moins une disposition affective spécifique qui ne se confond pas plus avec l'ennui qu'avec la culpabilité.

\section{NOTES}

1. J.-P. Sartre, B. Lévy, L'Espoir maintenant, Lagrasse, Verdier, 1991, p. 24.

2. J.-P. Sartre, L'Être et le néant, Paris, Gallimard, 2008, p. 66.

3. Nous retrouvons cette indétermination dans le texte d'Eschyle qui évoque l'angoisse à partir d'un terme très ordinaire signifiant crainte (deima). Cf. J.-J. Alrivie, «L'angoisse dans l'Agamemnon d'Eschyle à la lumière d'Être et temps de Martin Heidegger ", Philosophie, $\mathrm{N}^{\circ}$ 140, janvier 2019, p. 39.

4. J. Postel (sous la direction de), Dictionnaire de psychiatrie et de psychopathologie clinique, Paris, Larousse, 1993, p. 34.

5. M. Haar, Heidegger et l'essence de l'homme, Millon, Grenoble, 1990, p. 81.

6. C. Sommer, Heidegger, Aristote, Luther. Les sources aristotéliciennes et néo-testamentaires d'Être et temps, Paris, PUF, 2005, p. 171.

7. M. Heidegger, Prolégomènes à l'histoire du concept de temps, trad. par A. Boutot, Paris, Gallimard, 2006, p. 421. J. Greisch, Ontologie et temporalité, Paris, Gallimard, 1994, p. 236.

8. Selon Pascal, l'homme ne cesse de se masquer la misère de sa condition au moyen d'activités qui le "détourne" de la vue de sa misère : "Les hommes n'ayant pu guérir la mort, la misère, l'ignorance, ils se sont avisés, pour se rendre heureux, de n'y point penser », B. Pascal, Pensées, Euvres complètes, Paris, Seuil, 1963, fragment 133, p. 516.

9. P. Janet, De l'angoisse à l'extase, définit l'angoisse « comme une peur sans objet ». Cf. J. FavezBoutonier, L'Angoisse, Paris, PUF, 1949, p. 14.

10. Cf. M. Heidegger, Être et temps, trad. par F. Vezin, Paris, Gallimard, 1986, §30. Pour C. Sommer, cette opposition est principalement configurée par celle, néotestamentaire, entre crainte mondaine et servile (timor servilis) et crainte pure de Dieu (timor castus) ; C. Sommer, Heidegger, Aristote, Luther. Les sources aristotéliciennes et néo-testamentaires d'Être et temps, op. cit., p. 153.

11. B. Pascal, Pensées, fragment 201-206, p. 528. A. de Lamartine, "L'Isolement ", Cuvres poétiques, Paris, Gallimard, Bibliothèque de La Pléiade, 1963, p. 3 ; S. Freud, Inhibition, symptôme et angoisse, trad. par J. Doron et R. Doron, Paris, PUF, 1993, p. 51.

12. M. Heidegger, Être et temps, op. cit., §40, p. 238.

13. "L'angoisse n'est rien d'autre que l'expérience pure et simple de l'être au sens d'être-aumonde. Cette expérience peut, elle ne doit pas nécessairement - puisque toute possibilité d'être est placée sous l'égide d'un "pouvoir" - se rencontrer en un sens éminent dans la mort, plus exactement dans le mourir de la mort. Nous parlons alors de l'angoisse de la mort, qui est à distinguer radicalement de la peur de la mort ", M. Heidegger, Prolégomènes à l'histoire du concept de temps, p. 421.

14. M. Heidegger, Etre et temps, op. cit., §51.

15. La tâche la plus essentielle de la métaphysique depuis Platon est « de nous rappeler notre participation à l'éternel et de nous inviter à dépasser ainsi la contingence et la finitude de la vie individuelle », F. Dastur, La mort. Essai sur la finitude, Paris, Hatier, 1994.

16. M. Heidegger, Être et temps, op. cit., §50. 
17. M. Heidegger, Être et temps, op. cit., §68, p. 405 ; M. Haar, Heidegger et l'essence de l'homme, p. 8 ; F. Dastur, Heidegger et la question du temps, Paris, PUF, 1990, p. 52.

18. S. Kierkegaard, Le concept d'angoisse, Euvres complètes, t.7, Paris, Éditions de l'Orange, 1973, p. $144-145$ et p. 163.

19. C. Serban, Phénoménologie de la possibilité. Husserl et Heidegger, Paris, PUF, 2016, p. 128.

20. M. Heidegger, Être et temps, op. cit., p. 237.

21. J. Greisch, Ontologie et temporalité, op. cit., p. 234.

22. S. Kierkegaard, Le Concept d'angoisse, op. cit., p. 163. J. Wahl, Études kierkegaardiennes, Paris, Vrin, 1949, p. 220.

23. J.-P. Sartre, L'Être et le néant, op. cit., p. 64.

24. Dans sa postface de 1943 à Qu'est-ce que la métaphysique? Heidegger envisage le rien comme l'autre de l'étant et «le voile de l'être (Schleier des Seins) ». Cf. J.-L. Marion, Réduction et donation. Recherches sur Husserl, Heidegger et la phénoménologie, Paris, PUF, 1989, p. 274.

25. J. Lacan, Le Séminaire, Livre X, L'Angoisse, Paris, Le Seuil, 2004, (ce livre rassemble les leçons prononcées par Lacan au cours de l'année 1962-63). De manière bien peu convaincante, MarieClaude Lambotte envisage l'angoisse tant d'un point de vue existentiel (Kierkegaard, Heidegger) que d'un point de vue psychanalytique (Freud, Lacan) dans « Voir et savoir : vers une critique du concept d'angoisse" in Psychiatrie et existence, P. Fédida et J. Schotte (éds.), Grenoble, Millon, 1991, p. 281 et sq.

26. M. Boss, Psychanalyse et analytique du Dasein, trad. par Ph. Cabestan et F. Dastur, Paris, Vrin, 2007, p. 22.

27. J. Laplanche, Problématique I, L'Angoisse, Paris, PUF, 1980.

28. S. Freud, Introduction à la psychanalyse, trad. par S. Jankélévitch, Paris, Payot, 1974, ch. XVII, p. 379-380.

29. S. Freud, Trois essais sur la théorie de la sexualité, trad. par B. Reverchon-Jouve, Paris, Gallimard, 1980, p. 186, note de 1920.

30. S. Freud, Inhibition, symptôme et angoisse, trad. par J. et R. Doron, Paris, PUF, 1993, p. 24.

31. B. Kimura , Écrits de psychopathologie phénoménologiques, trad. par J. Bouderlique, Paris, PUF, 1992, p. 51.

32. L. Binswanger, «Sur la direction de recherche analytico-existentielle en psychiatrie » (1946), Analyse existentielle et psychanalyse freudienne. Discours, parcours, et Freud, trad. par R. Lewinter, Paris, Gallimard, 1970, p. 68-69.

33. Reprenant ce cas de phobie, Alice Holzhey-Kunz laisse curieusement de côté cette notion qui est pourtant au cœur de la tentative d'élucidation de Binswanger. A. Holzhey-Kunz, Introduction à la Daseinsanalyse. Un regard existential sur la souffrance psychique et sa thérapie, trad. par L. Defago, Argenteuil, Le Cercle herméneutique, 2016, p. 146 et sq.

34. J.-P. Sartre, L'Être et le néant, op. cit., p. 524.

35. L. Binswanger, "Sur la direction de recherche analytico-existentielle en psychiatrie ", in Analyse existentielle et psychanalyse freudienne. Discours, parcours, et Freud, op. cit., p. 61.

36. Ibid., p. 66.

37. Ibid., p. 70.

38. Binswanger rapproche cette angoisse du talon de l'angoisse du bouton décousu, qui ne tient plus qu'à un fil, ou encore de l'angoisse de la rupture du fil de salive, ou de l'évacuation des selles. Dans un cas comme dans l'autre nous retrouvons un même projet-de-monde restreint à la catégorie de la continuité. Ibid, p. 71-72.

39. Ibid., p. 72.

40. M. Heidegger, Qu'est-ce que la métaphysique? trad. par R. Munier, Cahier de L'Herne: Martin Heidegger, 1983, p. 51.

41. L. Binswanger, Le cas Lola Voss. Schizophrénie. Quatrième étude, trad. par Ph. Veysset, Paris, PUF, 2012, p. 57. 
42. Sur le sens de cette notion, cf. C. Romano, "Heidegger et l'achèvement de l'authenticité ", Être soi-même. Une autre histoire de la philosophie, Paris, Gallimard, 2019, p. 557-598.

43. Signalons que Heidegger, dans ses entretiens avec Medard Boss, récuse explicitement cette analyse de Binswanger à laquelle il reproche son caractère subjectiviste et formel. M. Heidegger, Séminaires de Zurich, trad. par C. Gros, Paris, Gallimard, 2010, p. 282-283.

44. J.-P. Sartre, La Transcendance de l'ego et autres textes phénoménologiques, Paris, Vrin, 2003, p. 128 et p. 196, note 99 .

45. J.-P. Sartre, La Transcendance de l'ego, op. cit., p. 80, note 74. Cette remarque de Sylvie Le Bon, fille adoptive de S. de Beauvoir, se trouve dans l'édition de ce texte paru en 1988.

46. Ph. Cabestan, "Spontanéité et captivité de la conscience ", in L'Être et la conscience. Recherches sur la psychologie et l'ontophénoménologie sartriennes, Bruxelles, éditions OUSIA, 2004, p. 125 et sq.

47. A. Kraus, "Existenzanalytische Aspekte der Raum-Phobien und ihrer Behandlung ", Jahrbuch für phänomenologische Anthropologie und Psychotherapie, 20-2004, p. 237-254.

48. L'émotion en tant que conduite est pour Sartre une forme de conscience qui, contrairement à une appréhension pragmatique du monde, le transforme magiquement : « dans l'émotion, c'est le corps qui, dirigé par la conscience change ses rapports au monde pour que le monde change ses qualités ", J.-P. Sartre, Esquisse d'une théorie des émotions, Paris, éditions Hermann, 2010, p. 44.

49. O. Dörr-Zegers, "Phénoménologie de l'angoisse », in La lecture du monde. Mélange en hommages à Yves Pelicier, J. Pélicier (éd.), Paris, PUF, 1998.

50. M. Zarader, Lire Être et temps, Paris, PUF, 2012, p.320-340.

51. M. Heidegger, Être et temps, §68, p.402-403. Nous retrouvons cette compréhension de l'angoisse selon la double modalité possible de l'authenticité et de l'inauthenticité dans la lecture par J.-J. Alrivie d'Agamemnon, qui oppose l'angoisse inauthentique du Chœur des Vieillards à l'angoisse authentique de Cassandre. J.-J. Alrivie, «L'angoisse dans l'Agamemnon d'Eschyle à la lumière d'Être et temps de Martin Heidegger ", in Philosophie, N 140 , op. cit., p. 49.

52. B. Kimura, «Le temps et l'angoisse », Écrits de psychopathologie phénoménologique, op. cit., p. 48-63. 